\title{
Dynamics of a System of Rational Higher-Order Difference Equation
}

\section{Banyat Sroysang}

Department of Mathematics and Statistics, Faculty of Science and Technology, Thammasat University, Pathumthani 12121, Thailand

Correspondence should be addressed to Banyat Sroysang; banyat@mathstat.sci.tu.ac.th

Received 5 February 2013; Accepted 22 April 2013

Academic Editor: Cengiz Çinar

Copyright (C) 2013 Banyat Sroysang. This is an open access article distributed under the Creative Commons Attribution License, which permits unrestricted use, distribution, and reproduction in any medium, provided the original work is properly cited.

We focus on a system of a rational $m$-order difference equation $x_{n+1}=\left(x_{n-m+1}\right) /\left(A+y_{n} y_{n-1} \cdots y_{n-m+1}\right), y_{n+1}=\left(y_{n-m+1}\right) /(B+$ $\left.x_{n} x_{n-1} \cdots x_{n-m+1}\right), n=0,1, \ldots$, where $A, B, x_{0}, x_{-1}, \ldots, x_{-m+1}, y_{0}, y_{-1}, \ldots, y_{-m+1} \in(0, \infty)$. We investigate the dynamical behavior of positive solution for the system.

\section{Introduction}

In 2011, Kurbanli et al. [1] studied the behavior of positive solutions of the system of rational difference equations

$$
\begin{aligned}
& x_{n+1}=\frac{x_{n-1}}{y_{n} x_{n-1}+1}, \\
& y_{n+1}=\frac{y_{n-1}}{x_{n} y_{n-1}+1},
\end{aligned}
$$

where the initial conditions are arbitrary nonnegative real numbers.

In the same year, Kurbanli [2] studied the behavior of solutions of the system of rational difference equations

$$
\begin{aligned}
& x_{n+1}=\frac{x_{n-1}}{y_{n} x_{n-1}-1}, \\
& y_{n+1}=\frac{y_{n-1}}{x_{n} y_{n-1}-1}, \\
& z_{n+1}=\frac{z_{n-1}}{y_{n} z_{n-1}-1},
\end{aligned}
$$

where the initial conditions are arbitrary real numbers. Moreover, Kurbanli [3] studied the behavior of the solutions of the difference equation system

$$
\begin{gathered}
x_{n+1}=\frac{x_{n-1}}{y_{n} x_{n-1}-1}, \\
y_{n+1}=\frac{y_{n-1}}{x_{n} y_{n-1}-1}, \\
z_{n+1}=\frac{1}{y_{n} z_{n}},
\end{gathered}
$$

where $x_{0}, x_{-1}, y_{0}, y_{-1}, z_{0}, z_{-1} \in \mathbb{R}$ such that $y_{0} x_{-1} \neq 1$, $x_{0} y_{-1} \neq 1$ and $y_{0} z_{0} \neq 1$.

In [4], Liu et al. gave more results of the solution of the system (2) including a new and simple expression of $z_{n}$ and the asymptotical behavior of the solution.

In [5], Stević showed that the system of difference equations

$$
\begin{array}{r}
x_{n+1}=\frac{a x_{n-1}}{b y_{n} x_{n-1}+c}, \quad y_{n+1}=\frac{\alpha y_{n-1}}{\beta x_{n} y_{n-1}+\gamma}, \\
n=0,1, \ldots,
\end{array}
$$

can be solved.

In 2012, Gu and Ding [6] derived two canonical state space forms from multiple-input multiple-output systems described by difference equations. 
The system of two nonlinear difference equations

$$
\begin{array}{r}
x_{n+1}=A+\frac{y_{n}}{x_{n-p}}, \quad y_{n+1}=A+\frac{x_{n}}{y_{n-q}}, \\
n=0,1, \ldots,
\end{array}
$$

was studied by Papaschinopoulos and Schinas [7], where $p, q \in \mathbb{N}$.

Moreover, the system of rational difference equations

$$
\begin{aligned}
x_{n+1}=\frac{x_{n}}{a+c y_{n}}, \quad y_{n+1} & =\frac{y_{n}}{b+d x_{n}}, \\
n & =0,1, \ldots,
\end{aligned}
$$

was studied by Clark et al. $[8,9]$, where $a, b, c, d \in(0, \infty)$ and $x_{0}, y_{0} \in[0, \infty)$.

Liu et al. [10] studied the behavior of a system of rational difference equations

$$
\begin{aligned}
& x_{n+1}=\frac{x_{n-1}}{y_{n} x_{n-1}-1}, \quad y_{n+1}=\frac{y_{n-1}}{x_{n} y_{n-1}-1}, \\
& z_{n+1}=\frac{1}{x_{n} z_{n-1}}, \quad n=0,1, \ldots,
\end{aligned}
$$

where the initial conditions are nonzero real numbers.

In 2012, Zhang et al. [11] studied the solutions, stability character, and asymptotic behavior of the system of a rational third-order difference equation

$$
\begin{array}{r}
x_{n+1}=\frac{x_{n-2}}{A+y_{n} y_{n-1} y_{n-2}}, \quad y_{n+1}=\frac{y_{n-2}}{B+x_{n} x_{n-1} x_{n-2}}, \\
n=0,1, \ldots,
\end{array}
$$

where $A, B, x_{0}, x_{-1}, x_{-2}, y_{0}, y_{-1}, y_{-2} \in(0, \infty)$.

In this paper, we studied the solutions, stability character, and asymptotic behavior of the system of a rational $m$-order difference equation

$$
\begin{aligned}
& x_{n+1}=\frac{x_{n-m+1}}{A+y_{n} y_{n-1} \cdots y_{n-m+1}}, \\
& y_{n+1}=\frac{y_{n-m+1}}{B+x_{n} x_{n-1} \cdots x_{n-m+1}}, \quad n=0,1, \ldots,
\end{aligned}
$$

where $A, B, x_{0}, x_{-1}, \ldots, x_{-m+1}, y_{0}, y_{-1}, \ldots, y_{-m+1} \in(0, \infty)$.

\section{Preliminaries}

Let $m \in \mathbb{N}$ and let $f: I_{x}^{m} \times I_{y}^{m} \rightarrow I_{x}$ and $g: I_{x}^{m} \times I_{y}^{m} \rightarrow I_{y}$ be continuously differentiable functions, where $I_{x}$ and $I_{y}$ are intervals in $\mathbb{R}$.

For any $\left(x_{0}, y_{0}\right),\left(x_{-1}, y_{-1}\right), \ldots,\left(x_{-m+1}, y_{-m+1}\right) \in I_{x} \times I_{y}$, the system of difference equations

$$
\begin{array}{r}
x_{n+1}=f\left(x_{n}, x_{n-1}, \ldots, x_{n-m+1}, y_{n}, y_{n-1}, \ldots, y_{n-m+1}\right), \\
y_{n+1}=g\left(x_{n}, x_{n-1}, \ldots, x_{n-m+1}, y_{n}, y_{n-1}, \ldots, y_{n-m+1}\right), \\
n=0,1, \ldots,
\end{array}
$$

has a unique solution $\left\{\left(x_{n}, y_{n}\right)\right\}_{n=-m+1}^{\infty}$.
Definition 1. A point $(\bar{x}, \bar{y}) \in I_{x} \times I_{y}$ is called an equilibrium point of the system (10) if $\bar{x}=f(\bar{x}, \bar{x}, \ldots, \bar{x}, \bar{y}, \bar{y}, \ldots, \bar{y})$ and $\bar{y}=g(\bar{x}, \bar{x}, \bar{x}, \ldots, \bar{y}, \bar{y}, \ldots, \bar{y})$.

Definition 2. The linearized system of the system (10) about the equilibrium $(\bar{x}, \bar{y})$ is the system of linear difference equations

$$
\begin{aligned}
x_{n+1}= & \sum_{i=0}^{m-1}\left(\frac{\partial f(\bar{x}, \bar{x}, \ldots, \bar{x}, \bar{y}, \bar{y}, \ldots, \bar{y})}{\partial x_{n-i}} x_{n-i}\right. \\
& \left.+\frac{\partial f(\bar{x}, \bar{x}, \ldots, \bar{x}, \bar{y}, \bar{y}, \ldots, \bar{y})}{\partial y_{n-i}} y_{n-i}\right), \\
y_{n+1}=\sum_{i=0}^{m-1}\left(\frac{\partial g(\bar{x}, \bar{x}, \ldots, \bar{x}, \bar{y}, \bar{y}, \ldots, \bar{y})}{\partial x_{n-i}} x_{n-i}\right. & \left.\frac{\partial g(\bar{x}, \bar{x}, \ldots, \bar{x}, \bar{y}, \bar{y}, \ldots, \bar{y})}{\partial y_{n-i}} y_{n-i}\right) .
\end{aligned}
$$

Definition 3. An equilibrium point $(\bar{x}, \bar{y})$ of the system (10) is said to be stable relative to $I_{x} \times I_{y}$ if for every $\epsilon>0$, there exists $\delta>0$ such that for any $\left(x_{0}, y_{0}\right),\left(x_{-1}, y_{-1}\right), \ldots$, $\left(x_{-m+1}, y_{-m+1}\right) \in I_{x} \times I_{y}$, with

$$
\max \left\{\sum_{i=-m+1}^{0}\left|x_{i}-\bar{x}\right|, \sum_{i=-m+1}^{0}\left|y_{i}-\bar{y}\right|\right\}<\delta .
$$

One has $\max \left\{\left|x_{n}-\bar{x}\right|,\left|y_{n}-\bar{y}\right|\right\}<\epsilon$ for all $n \geq-m+1$.

Definition 4. An equilibrium point $(\bar{x}, \bar{y})$ of the system (10) is called an attractor relative to $I_{x} \times I_{y}$ if for all $\left(x_{0}, y_{0}\right),\left(x_{-1}, y_{-1}\right), \ldots,\left(x_{-m+1}, y_{-m+1}\right) \in I_{x} \times I_{y}$, one has $\lim _{n \rightarrow \infty} x_{n}=\bar{x}$ and $\lim _{n \rightarrow \infty} y_{n}=\bar{y}$.

Definition 5. An equilibrium point $(\bar{x}, \bar{y})$ of the system (10) is said to be asymptotically stable relative to $I_{x} \times I_{y}$ if it is stable, and it is also an attractor.

Definition 6. An equilibrium point $(\bar{x}, \bar{y})$ of the system (10) is said to be unstable if it is not stable.

Theorem 7 (see [12]). Let $X(n+1)=F(X(n)), n=0,1, \ldots$, be a system of difference equations and let $\bar{X}$ be the equilibrium point of the system. If all eigenvalues of the Jacobian matrix evaluated at $\bar{X}$ lie inside the open unit disk, then $\bar{X}$ is asymptotically stable. If one of them has a modulus greater than one, then $\bar{X}$ is unstable.

Theorem 8 (see [13]). Let $X(n+1)=F(X(n)), n=0,1, \ldots$, be a system of difference equations and let $\bar{X}$ be the equilibrium point of the system. Assume that the characteristic polynomial of the system about $\bar{X}$ is $a_{0} \lambda^{n}+a_{1} \lambda^{n-1}+\cdots+a_{n-1} \lambda+a_{n}$ where $a_{i} \in \mathbb{R}$ for all $i$ and $a_{0}>0$. Then all roots of the characteristic equation lie inside the open unit disk if and only if $\Delta_{k}>0$ for 
all positive integer $k \leq n$, where $\Delta_{k}$ is the principal minor of order $k$ of the $n \times n$ matrix

$$
\Delta_{n}=\left(\begin{array}{ccccc}
a_{1} & a_{3} & a_{5} & \cdots & 0 \\
a_{0} & a_{2} & a_{4} & \cdots & 0 \\
0 & a_{1} & a_{3} & \cdots & 0 \\
\vdots & \vdots & \vdots & \ddots & \vdots \\
0 & 0 & 0 & \cdots & a_{n}
\end{array}\right)
$$

\section{Results}

We note that

(i) if $A<1$ and $B<1$ then the system (9) has equilibrium $(0,0)$ and $(\sqrt[m]{1-B}, \sqrt[m]{1-A})$

(ii) if $A=1$ and $B<1$ then the system (9) has equilibrium $(0,0)$ and $(\sqrt[m]{1-B}, 0)$

(iii) if $A<1$ and $B=1$ then the system (9) has equilibrium $(0,0)$ and $(0, \sqrt[m]{1-A})$

(iv) if $A>1$ and $B>1$ then $(0,0)$ is the unique equilibrium point of the system (9).
Theorem 9. Let $\left(x_{n}, y_{n}\right)$ be positive solution of the system (9). For all nonnegative integer $k$, one has

$$
0 \leq x_{n} \leq \begin{cases}\frac{x_{-m+1}}{A^{k+1}}, & n=m k+1 ; \\ \frac{x_{-m+2}}{A^{k+1}}, & n=m k+2 ; \\ \vdots & \\ \frac{x_{0}}{A^{k+1}}, & n=m k+m,\end{cases}
$$

$$
0 \leq y_{n} \leq \begin{cases}\frac{y_{-m+1}}{B^{k+1}}, & n=m k+1 ; \\ \frac{y_{-m+2}}{B^{k+1}}, & n=m k+2 ; \\ \vdots & \\ \frac{y_{0}}{B^{k+1}}, & n=m k+m .\end{cases}
$$

Proof. Obviously, they are true for $k=0$. Suppose that they are true for $k=l$. Then

$$
x_{n}= \begin{cases}x_{m(l+1)+1} \leq \frac{x_{m(l+1)-m+1}}{A}=\frac{1}{A} x_{m l+1} \leq \frac{1}{A}\left(\frac{x_{-m+1}}{A^{l+1}}\right), & n=m(l+1)+1 ; \\ x_{m(m+1)+2} \leq \frac{x_{m(l+1)-m+2}}{A}=\frac{1}{A} x_{m l+2} \leq \frac{1}{A}\left(\frac{x_{-m+2}}{A^{l+1}}\right), & n=m(l+1)+2 ; \\ \vdots & y_{n}= \begin{cases}x_{m(l+1)+m} \leq \frac{x_{m(l+1)}}{A}=\frac{1}{A} x_{m l+m} \leq \frac{1}{A}\left(\frac{x_{0}}{A^{l+1}}\right), & n=m(l+1)+m, \\ y_{m(l+1)+2} \leq \frac{y_{m(l+1)-m+2}}{B}=\frac{1}{B} y_{m l+2} \leq \frac{1}{B}\left(\frac{y_{-m+2}}{B^{l+1}}\right), & n=4(m+1)+2 ; \\ \vdots & n=m(l+1)+1 ; \\ y_{m(l+1)+m} \leq \frac{y_{m(l+1)}}{B}=\frac{1}{B} y_{m l+m} \leq \frac{1}{B}\left(\frac{y_{0}}{B^{l+1}}\right), & n=m(l+1)+m .\end{cases} \end{cases}
$$

Thus, they are true for $k=l+1$.

By the mathematical induction, this proof is completed.

Corollary 10. Let $\left(x_{n}, y_{n}\right)$ be positive solution of the system (9). If $A>1$ and $B>1$, then the sequence $\left\{\left(x_{n}, y_{n}\right)\right\}$ converges exponentially to the equilibrium point $(0,0)$.

Theorem 11. Let $A>1$ and $B>1$. Then the equilibrium point $(0,0)$ of the system $(9)$ is asymptotically stable.

Proof. The linearized system of the system (9) about the equilibrium $(0,0)$ is

$$
\Phi_{n+1}=D \Phi_{n}
$$

where

$$
\Phi_{n}=\left(\begin{array}{c}
x_{n} \\
x_{n-1} \\
x_{n-2} \\
\vdots \\
x_{n-m+1} \\
y_{n} \\
y_{n-1} \\
y_{n-2} \\
\vdots \\
y_{n-m+1}
\end{array}\right)
$$




$$
D=\left(\begin{array}{cccccccccc}
0 & 0 & \cdots & 0 & \frac{1}{A} & 0 & 0 & \cdots & 0 & 0 \\
1 & 0 & \cdots & 0 & 0 & 0 & 0 & \cdots & 0 & 0 \\
0 & 1 & \cdots & 0 & 0 & 0 & 0 & \cdots & 0 & 0 \\
\vdots & \ddots & \ddots & \ddots & \ddots & \ddots & \ddots & \vdots & \vdots & \vdots \\
0 & 0 & \cdots & 1 & 0 & 0 & 0 & 0 & 0 & 0 \\
0 & 0 & \cdots & 0 & 0 & 0 & 0 & 0 & 0 & \frac{1}{B} \\
0 & 0 & \cdots & 0 & 0 & 1 & 0 & 0 & 0 & 0 \\
0 & 0 & \cdots & 0 & 0 & 0 & 1 & 0 & 0 & 0 \\
\vdots & \ddots & \ddots & \ddots & \ddots & \ddots & \ddots & \ddots & \ddots & \vdots \\
0 & 0 & \cdots & 0 & 0 & 0 & 0 & 0 & 1 & 0
\end{array}\right)
$$

The characteristic equation of the system (16) is

$$
\left(\lambda^{m}-\frac{1}{A}\right)\left(\lambda^{m}-\frac{1}{B}\right)=0
$$

Thus, $|\lambda|<1$. By Theorem 7 , the equilibrium point $(0,0)$ is asymptotically stable.

Theorem 12. Let $A<1$ and $B<1$. Then both the equilibrium points $(0,0)$ and $(\sqrt[m]{1-B}, \sqrt[m]{1-A})$ of the system (9) are unstable.

Proof. We note by the characteristic equation (18) that $|\lambda|>$ 1 and then, by Theorem 7 , the equilibrium point $(0,0)$ is unstable.

Next, we consider the equilibrium point $(\sqrt[m]{1-B}$, $\sqrt[m]{1-A})$. The linearized system of the system (9) about the equilibrium $(\sqrt[m]{1-B}, \sqrt[m]{1-A})$ is

$$
\Phi_{n+1}=G \Phi_{n}
$$

where

$$
\Phi_{n}=\left(\begin{array}{c}
x_{n} \\
x_{n-1} \\
x_{n-2} \\
\vdots \\
x_{n-m+1} \\
y_{n} \\
y_{n-1} \\
y_{n-2} \\
\vdots \\
y_{n-m+1}
\end{array}\right) \text {, }
$$

$$
G=\left(\begin{array}{cccccccccc}
0 & 0 & \cdots & 0 & 1 & \alpha & \alpha & \cdots & \alpha & \alpha \\
1 & 0 & \cdots & 0 & 0 & 0 & 0 & \cdots & 0 & 0 \\
0 & 1 & \cdots & 0 & 0 & 0 & 0 & \cdots & 0 & 0 \\
\vdots & \ddots & \ddots & \ddots & \ddots & \ddots & \ddots & \vdots & \vdots & \vdots \\
0 & 0 & \cdots & 1 & 0 & 0 & 0 & 0 & 0 & 0 \\
\beta & \beta & \cdots & \beta & \beta & 0 & 0 & 0 & 0 & 1 \\
0 & 0 & \cdots & 0 & 0 & 1 & 0 & 0 & 0 & 0 \\
0 & 0 & \cdots & 0 & 0 & 0 & 1 & 0 & 0 & 0 \\
\vdots & \ddots & \ddots & \ddots & \ddots & \ddots & \ddots & \ddots & \ddots & \vdots \\
0 & 0 & \cdots & 0 & 0 & 0 & 0 & 0 & 1 & 0
\end{array}\right),
$$

in which

$$
\begin{aligned}
& \alpha=-\sqrt[m]{(1-A)^{m-1}(1-B)}, \\
& \beta=-\sqrt[m]{(1-A)(1-B)^{m-1}} .
\end{aligned}
$$

The characteristic polynomial of the system (19) is

$$
\begin{array}{r}
1-\alpha \beta-\sum_{i=1}^{m}\left(i \alpha \beta \lambda^{i-1}\right)-2 \lambda^{m} \\
-\sum_{i=1}^{m-1}\left(i \alpha \beta \lambda^{2 m-(i+1)}\right)+\lambda^{2 m} .
\end{array}
$$

We note the characteristic polynomial $a_{0} \lambda^{2 m}+a_{1} \lambda^{2 m-1}+$ $\cdots+a_{2 m-1} \lambda+a_{2 m}$ that $a_{1}=0$. Thus, we obtain that not all of $\Delta_{k}>0, k=1,2, \ldots, 2 m$. By Theorems 7 and 8 , the equilibrium point $(\sqrt[m]{1-B}, \sqrt[m]{1-A})$ is unstable.

Theorem 13. Let $A, B<1$ and $\Omega_{1}=(0, \sqrt[m]{1-B}) \times(\sqrt[m]{1-A}$, $\infty), \Omega_{2}=(\sqrt[m]{1-B}, \infty) \times(0, \sqrt[m]{1-A})$. Assume that $\left\{\left(x_{n}\right.\right.$, $\left.\left.y_{n}\right)\right\}_{n=-m+1}^{\infty}$ satisfies the system (9). Then

(i) if $\left\{\left(x_{n}, y_{n}\right)\right\}_{n=-m+1}^{0} \subseteq \Omega_{1}$, then $\left\{\left(x_{n}, y_{n}\right)\right\}_{n=-m+1}^{\infty} \subseteq \Omega_{1}$;

(ii) if $\left\{\left(x_{n}, y_{n}\right)\right\}_{n=-m+1}^{0} \subseteq \Omega_{2}$, then $\left\{\left(x_{n}, y_{n}\right)\right\}_{n=-m+1}^{\infty} \subseteq \Omega_{2}$.

Proof. (i) Assume that $\left\{\left(x_{n}, y_{n}\right)\right\}_{n=-m+1}^{0} \subseteq \Omega_{1}$. Then, for any $i \in\{0,1, \ldots, m-1\}$,

$$
\begin{aligned}
& x_{i+1}=\frac{x_{i-m+1}}{A+y_{i} y_{i-1} \cdots y_{i-m+1}}<\frac{x_{i-m+1}}{A+(\sqrt[m]{1-A})^{m}}=x_{i-m+1} \\
& y_{i+1}=\frac{y_{i-m+1}}{B+x_{i} x_{i-1} \cdots x_{i-m+1}}>\frac{y_{i-m+1}}{B+(\sqrt[m]{1-B})^{m}}=y_{i-m+1}
\end{aligned}
$$

Then $\left(x_{1}, y_{1}\right),\left(x_{2}, y_{2}\right), \ldots,\left(x_{m}, y_{m}\right) \in \Omega_{1}$. 
Next, we suppose that $\left(x_{k}, y_{k}\right),\left(x_{k-1}, y_{k-1}\right), \ldots,\left(x_{k-m+1}\right.$, $\left.y_{k-m+1}\right) \in \Omega_{1}$ where $k$ is a positive integer. Then

$$
\begin{aligned}
& x_{k+1}=\frac{x_{k-m+1}}{A+y_{k} y_{k-1} \cdots y_{k-m+1}}<\frac{x_{k-m+1}}{A+(\sqrt[m]{1-A})^{m}}=x_{k-m+1}, \\
& y_{k+1}=\frac{y_{k-m+1}}{B+x_{k} x_{k-1} \cdots x_{k-m+1}}>\frac{y_{k-m+1}}{B+(\sqrt[m]{1-B})^{m}}=y_{k-m+1} .
\end{aligned}
$$

Then $\left(x_{k+1}, y_{k+1}\right) \in \Omega_{1}$.

By the mathematical induction, $\left\{\left(x_{n}, y_{n}\right)\right\}_{n=-m+1}^{\infty} \subseteq \Omega_{1}$.

(ii) This is similar to the proof of (i).

\section{Acknowledgment}

The author would like to thank the referees for their useful comments and suggestions.

\section{References}

[1] A. S. Kurbanli, C. Çinar, and I. Yalçinkaya, "On the behavior of positive solutions of the system of rational difference equations $x_{n+1}=x_{n-1} /\left(y_{n} x_{n-1}+1\right), y_{n+1}=y_{n-1} /\left(x_{n} y_{n-1}+1\right)$," Mathematical and Computer Modelling, vol. 53, no. 5-6, pp. 1261-1267, 2011.

[2] A. S. Kurbanli, "On the behavior of solutions of the system of rational difference equations: $x_{n+1}=x_{n-1} /\left(y_{n} x_{n-1}-1\right), y_{n+1}=$ $y_{n-1} /\left(x_{n} y_{n-1}-1\right)$, and $z_{n+1}=z_{n-1} /\left(y_{n} z_{n-1}-1\right)$," Discrete Dynamics in Nature and Society, vol. 2011, Article ID 932362, 12 pages, 2011.

[3] A. S. Kurbanli, "On the behavior of solutions of the system of rational difference equations: $x_{n+1}=x_{n-1} /\left(y_{n} x_{n-1}-1\right), y_{n+1}=$ $y_{n-1} /\left(x_{n} y_{n-1}-1\right)$, and $z_{n+1}=z_{n-1} /\left(y_{n} z_{n-1}-1\right), "$ Discrete Dynamics in Nature and Society, vol. 2011, Article ID 932362, 12 pages, 2011.

[4] K. Liu, Z. Zhao, X. Li, and P. Li, "More on three-dimensional systems of rational difference equations," Discrete Dynamics in Nature and Society, vol. 2011, Article ID 178483, 9 pages, 2011.

[5] S. Stević, "On a system of difference equations," Applied Mathematics and Computation, vol. 218, no. 7, pp. 3372-3378, 2011.

[6] Y. Gu and R. Ding, "Observable state space realizations for multivariable systems," Computers \& Mathematics with Applications, vol. 63, no. 9, pp. 1389-1399, 2012.

[7] G. Papaschinopoulos and C. J. Schinas, "On a system of two nonlinear difference equations," Journal of Mathematical Analysis and Applications, vol. 219, no. 2, pp. 415-426, 1998.

[8] D. Clark and M. R. S. Kulenović, "A coupled system of rational difference equations," Computers \& Mathematics with Applications, vol. 43, no. 6-7, pp. 849-867, 2002.

[9] D. Clark, M. R. S. Kulenović, and J. F. Selgrade, "Global asymptotic behavior of a two-dimensional difference equation modelling competition," Nonlinear Analysis: Theory, Methods \& Applications, vol. 52, no. 7, pp. 1765-1776, 2003.

[10] K. Liu, Z. Wei, P. Li, and W. Zhong, "On the behavior of a system of rational difference equations $x_{n+1}=x_{n-1} /\left(y_{n} x_{n-1}-1\right), y_{n+1}=$ $y_{n-1} /\left(x_{n} y_{n-1}-1\right), z_{n+1}=1 / x_{n} z_{n-1}$," Discrete Dynamics in Nature and Society, vol. 2012, Article ID 105496, 9 pages, 2012.

[11] Q. Zhang, L. Yang, and J. Liu, "Dynamics of a system of rational third-order difference equation," Advances in Difference Equations, vol. 2012, article 136, p. 6, 2012.
[12] H. Sedaghat, Nonlinear Difference Equations, vol. 15 of Mathematical Modelling: Theory and Applications, Kluwer Academic Publishers, Dordrecht, The Netherlands, 2003.

[13] V. L. Kocić and G. Ladas, Global Behavior of Nonlinear Difference Equations of Higher Order with Applications, vol. 256 of Mathematics and Its Applications, Kluwer Academic Publishers, Dordrecht, The Netherlands, 1993. 


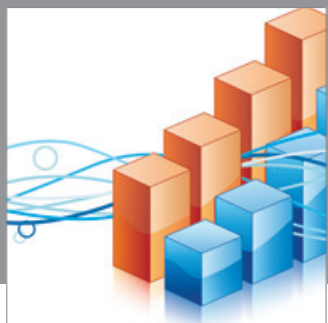

Advances in

Operations Research

mansans

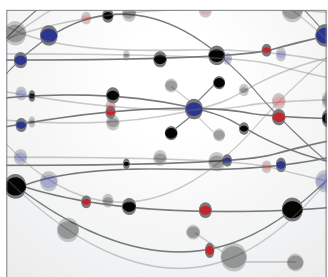

The Scientific World Journal
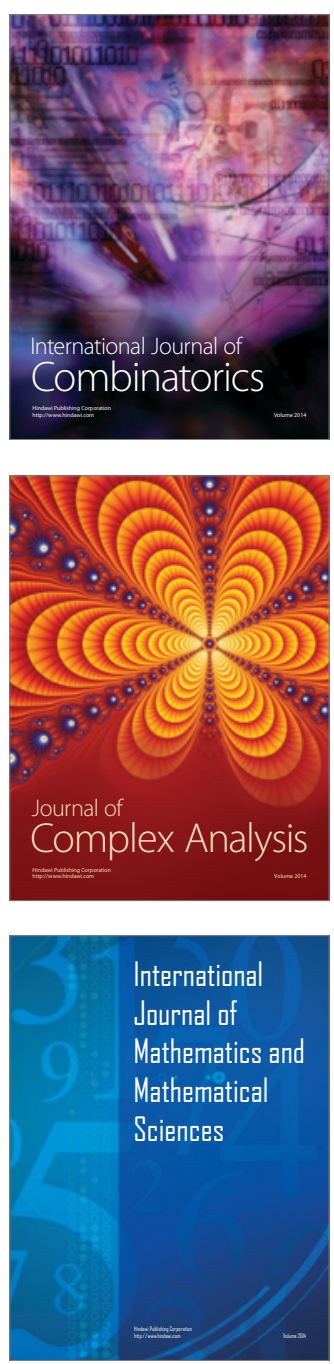
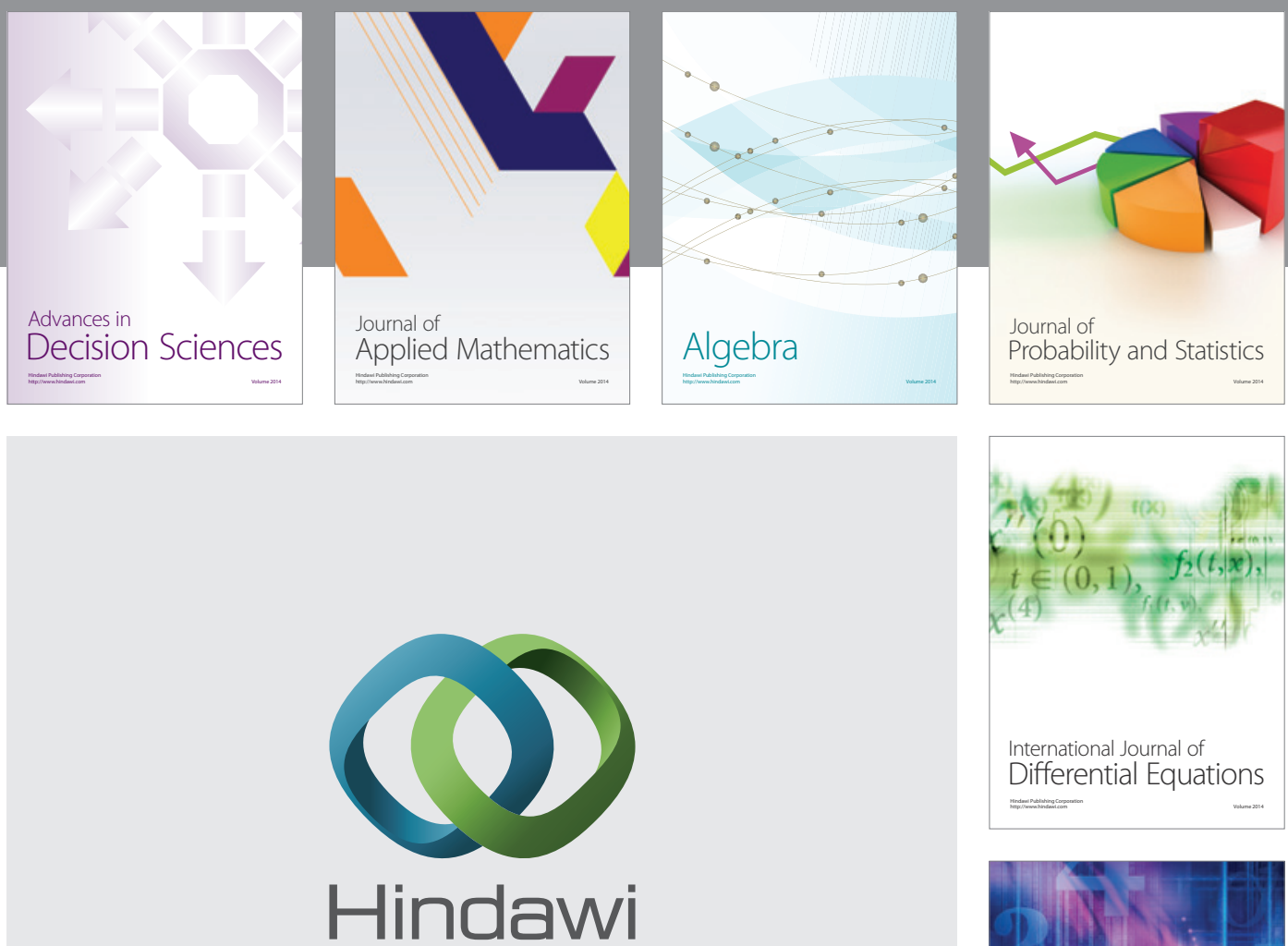

Submit your manuscripts at http://www.hindawi.com
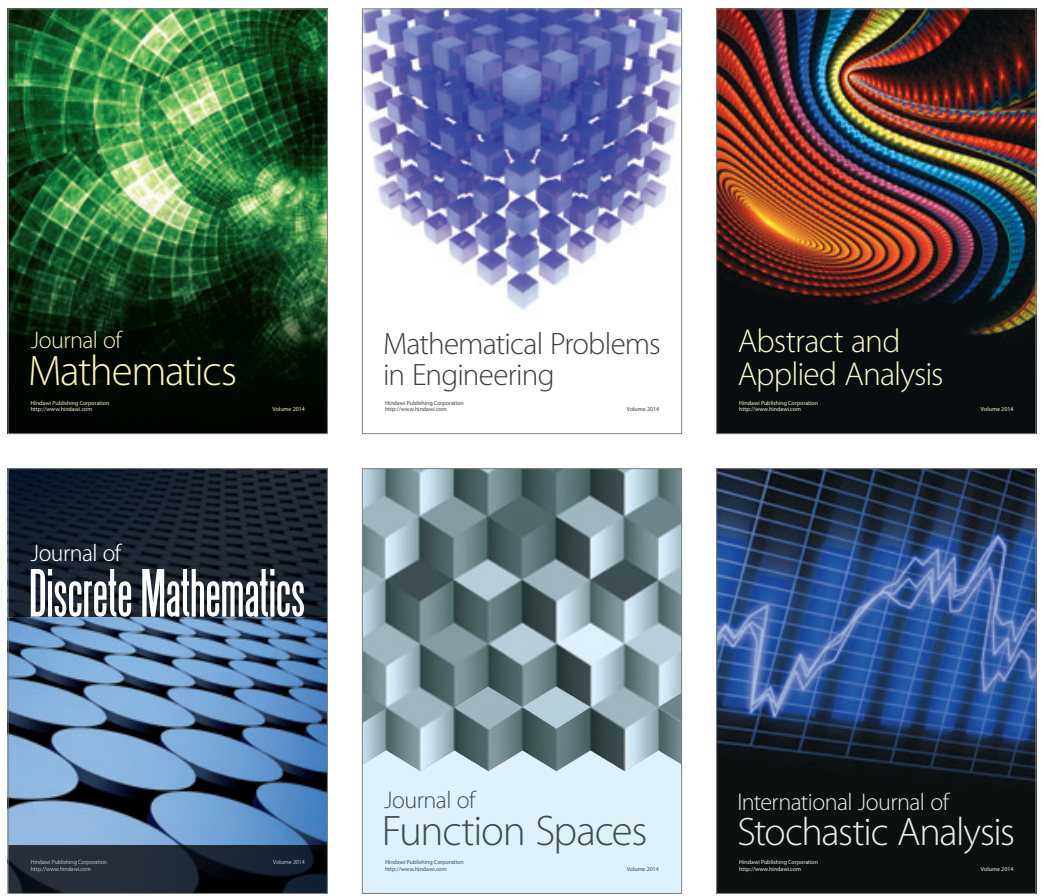

Journal of

Function Spaces

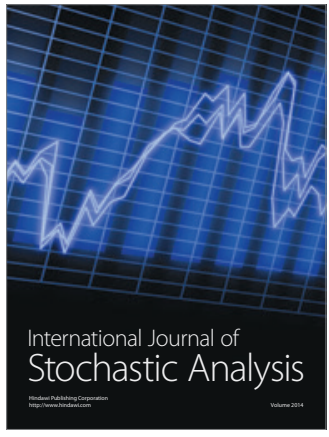

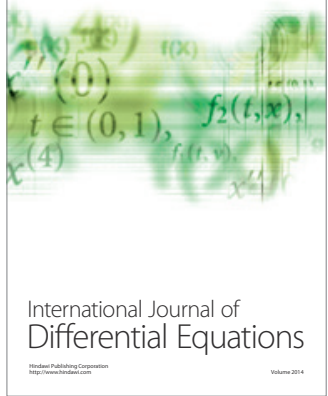
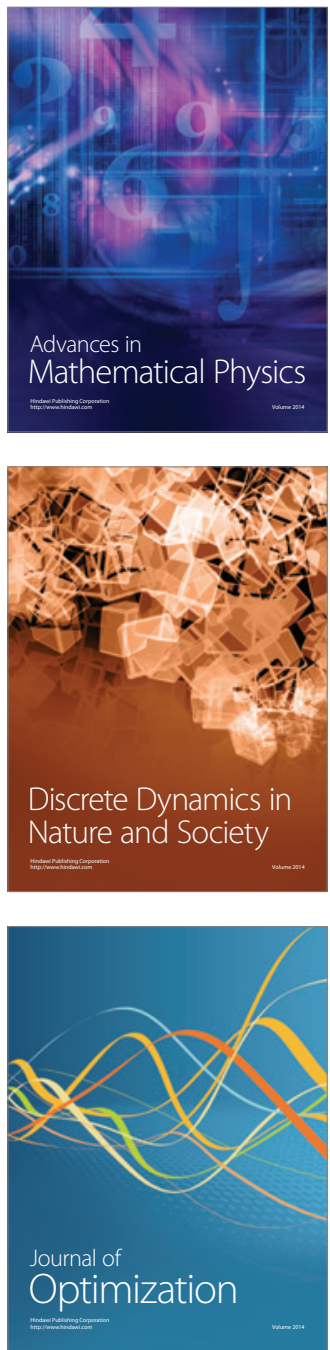